\title{
Neurally-Adjusted Ventilatory Assist Can Facilitate Extubation in Neonates With Congenital Diaphragmatic Hernia
}

\author{
Ryan D Meinen, Yousef I Alali, Awni Al-Subu, Michael Wilhelm, Catherine L Wraight, \\ Ryan M McAdams, Jamie J Limjoco, and David J McCulley
}

\begin{abstract}
BACKGROUND: Congenital diaphragmatic hernia is associated with a high risk of neonatal mortality and long-term morbidity due to lung hypoplasia, pulmonary hypertension, and prolonged exposure to positive-pressure ventilation. Ventilator-associated lung injury may be reduced by using approaches that facilitate the transition from invasive ventilation to noninvasive ventilation (NIV), such as with neurally-adjusted ventilatory assist (NAVA). We reported our use of NAVA in neonatal patients with congenital diaphragmatic hernia during the transition from invasive ventilation to NIV. METHODS: A retrospective analysis of neonatal subjects with congenital diaphragmatic hernia admitted to a tertiary care children's hospital between December 2015 and May 2018 was conducted. Subject data and factors that affected the use of NAVA were analyzed. RESULTS: Ten neonatal subjects with congenital diaphragmatic hernia were placed on NAVA, and 6 were successfully transitioned, after surgery, from pressure control synchronized intermittent mandatory ventilation to invasive ventilation with NAVA and then to NIV with NAVA without the need for re-intubation. The transition from pressure control synchronized intermittent mandatory ventilation to invasive ventilation with NAVA resulted in a decrease in peak inspiratory pressure, mean airway pressure, and $\mathrm{F}_{\mathrm{IO}_{2}}$. Barriers to the use of NAVA included symptomatic pleural effusion or chylothorax and pulmonary sequestration. CONCLUSIONS: Both invasive ventilation with NAVA and NIV with NAVA were used successfully in subjects with congenital diaphragmatic hernia during the transition from invasive ventilation to NIV. The transition to NAVA was associated with a decrease in peak inspiratory pressure, mean airway pressure, and the need for supplemental oxygen. A prospective trial is needed to determine the short- and long-term impacts of this mode of ventilation in neonates with congenital diaphragmatic hernia. Key words: Neurally adjusted ventilatory assist; NAVA; NIVNAVA; congenital diaphragmatic hernia; ventilation; ventilator-associated lung injury. [Respir Care 2021;66(1):41-49. (C) 2021 Daedalus Enterprises]
\end{abstract}

\section{Introduction}

Congenital diaphragmatic hernia $(\mathrm{CDH})$ is a common and severe birth defect associated with significant morbidity and mortality due to lung hypoplasia and pulmonary hypertension. ${ }^{1}$ Patients with $\mathrm{CDH}$ require positive-pressure

Drs Meinen, Wraight, McAdams, Limjoco, and McCulley are affiliated with the Division of Neonatology, University of Wisconsin-Madison, Madison, Wisconsin. Mr Alali is affiliated with the Division of Respiratory Therapy, University of Wisconsin-Madison, Madison, Wisconsin. Drs Al-Subu and Wilhelm are affiliated with the Division of Critical Care Medicine, Department of Pediatrics, University of Wisconsin-Madison, Madison, Wisconsin. ventilation (mechanical ventilation) after birth; however, the use of mechanical ventilation in these patients may lead to injury of the hypoplastic lungs and impair postnatal lung development. $^{2}$ To reduce ventilator-associated lung injury, identifying a mode of ventilation that minimizes barotrauma and permits rapid weaning by synchronizing with spontaneous breathing is essential. Neurally-adjusted

\footnotetext{
Drs Meinen and McCulley presented a version of this paper at the Pediatric Critical Care Regional Conference, held May 23, 2018, in Madison, Wisconsin, and at the Midwest Society of Pediatric Research meeting, held October 2, 2018, in Royal Oak, Michigan.
}

The authors have disclosed no conflicts of interest. 


\section{NAVA to Facilitate Extubation in NeOnates With CDH}

ventilatory assist (NAVA) is a mode of ventilation that has been demonstrated to synchronize with spontaneous breathing in both neonates and children who are intubated and those not intubated. ${ }^{3,4}$

Until recently, NAVA use in patients with $\mathrm{CDH}$ has been uncommon because it requires continuous assessment of diaphragmatic activity. Two recent case series ${ }^{5,6}$ demonstrate the use of invasive ventilation with NAVA, and one case series ${ }^{7}$ demonstrates the use of noninvasive ventilation (NIV) with NAVA (NIV-NAVA) in neonates with $\mathrm{CDH}^{5-7}$ In our study, we demonstrated that NAVA can be used during the transition from invasive ventilation to NIV in neonates with $\mathrm{CDH}$ after surgical repair of the diaphragm. We reported that the transition from pressure control synchronized intermittent mandatory ventilation to invasive ventilation with NAVA was associated with a decrease in peak inspiratory pressure (PIP), mean airway pressure $\left(\overline{\mathrm{P}}_{\mathrm{aw}}\right)$, and $\mathrm{F}_{\mathrm{IO}_{2}}$. Furthermore, we identified common barriers to the successful use of NAVA in neonates with CDH to better guide the future use of NAVA in this patient population.

\section{Methods}

This study was approved by the University of Wisconsin School of Medicine and Public Health Institutional Review Board. A retrospective analysis was performed and included all neonatal patients admitted to the pediatric or neonatal intensive care units at the American Family Children's Hospital from December 2015 through May 2018 for surgical repair of CDH. This analysis followed the implementation of a $\mathrm{CDH}$ management guideline that recommended the use of NAVA during the postoperative weaning phase of respiratory support (Fig. 1). Although some patients with CDH may not require invasive PPV after surgical repair, the approach followed during the study period included use of pressure control synchronized intermittent mandatory ventilation (PC-SIMV) from birth through the immediate postoperative phase of care. The transition from PC-SIMV to invasive ventilation with NAVA was attempted 24-72 $\mathrm{h}$ after $\mathrm{CDH}$ repair and the subjects were subsequently extubated to NIV-NAVA. If the subjects tolerated this transition, then they were further weaned to nasal CPAP, high-flow nasal cannula, or room air. If the transition from PC-SIMV to invasive ventilation with NAVA was not successful, then the subject was managed by using PC-SIMV until extubation to avoid

Correspondence: David J McCulley MD, Division of Neonatology, Department of Pediatrics, School of Medicine and Public Health, University of Wisconsin-Madison, 408 McConnell Hall, 1010 Mound Street, Madison, WI 52726. E-mail: dmcculley@wisc.edu.

DOI: $10.4187 /$ respcare.07681

\section{QUICK LOOK}

\section{Current knowledge}

Neonates with congenital diaphragmatic hernia (CDH) have a significant risk of morbidity and mortality due to lung hypoplasia and pulmonary hypertension. To improve the outcome for neonates with $\mathrm{CDH}$, a primary objective is to reduce their exposure to positivepressure ventilation and to avoid ventilator-associated lung injury. Neurally-adjusted ventilatory assist (NAVA) has been demonstrated to improve patient-ventilator synchrony in both neonatal and pediatric patients.

\section{What this paper contributes to our knowledge}

NAVA was successfully used during the transition from invasive to noninvasive ventilation in subjects with $\mathrm{CDH}$. The transition from conventional mechanical ventilation to NAVA resulted in decreased peak inspiratory pressure, mean airway pressure, and need for supplemental oxygen. Common barriers that limited the use of NAVA in subjects with CDH included symptomatic pleural effusion and chylothorax.

prolonging invasive ventilation. Further weaning was carried out until the subjects were off all respiratory support or on nasal cannula oxygen that was acceptable for home use.

According to the $\mathrm{CDH}$ management guideline, all respiratory care decisions were at the discretion of the attending physician. Two years before the development of this $\mathrm{CDH}$ guideline, our group began using NAVA routinely in postoperative neonatal patients and in infants born preterm. Although the $\mathrm{CDH}$ guideline does not discuss specific NAVA parameters for patients with $\mathrm{CDH}$, our group generally targets electrical activity of the diaphragm $\left(\mathrm{EA}_{\mathrm{di}}\right)$ peaks in the range of 5-15 $\mu \mathrm{V}$ while adjusting the NAVA level accordingly and monitoring work of breathing and oxygen requirement. The target $\mathrm{EA}_{\mathrm{di}}$ minimum is $<2 \mu \mathrm{V}$, and PEEP is increased if the $\mathrm{EA}_{\mathrm{di}}$ minimum is persistently $>2$ $\mu \mathrm{V}$ or if there is increased work of breathing or a need for supplemental oxygen.

Electronic medical records and data from the Children's Hospital Neonatal Consortium Database were queried to gather subject demographic information, the side and type of $\mathrm{CDH}$, the surgical approach, the type of respiratory support, and information with regard to associated complications. Demographic information included gestational age at birth, birth weight, sex, prenatal lung measurements, type of surgical repair, presence of additional anatomic anomalies or chromosome abnormalities, and whether pulmonary hypertension that required treatment 


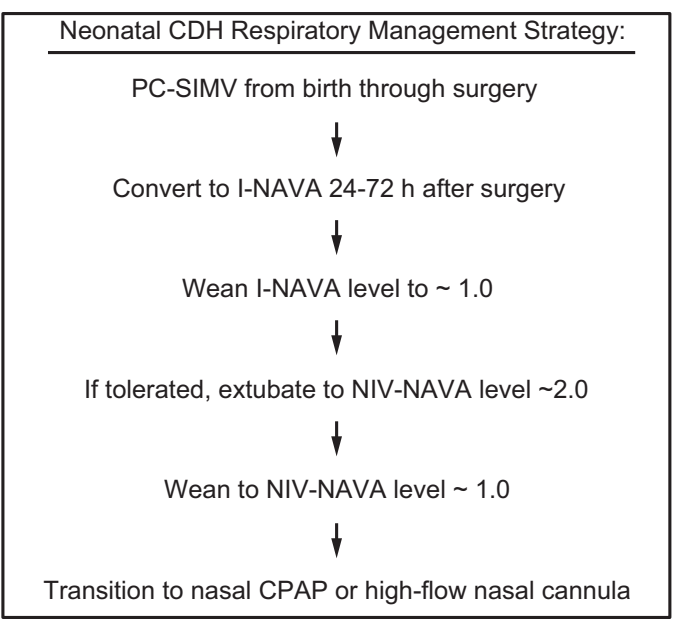

Fig. 1. Standard respiratory management approach included in the American Family Children's Hospital, University of WisconsinMadison, Clinical Care Guideline for neonates with congenital diaphragmatic hernia (CDH). PC-SIMV = pressure control synchronized intermittent mechanical ventilation, I-NAVA = invasive neurallyadjusted ventilatory assist, NIV-NAVA = noninvasive NAVA.

with pulmonary vasodilator medications was present during the first $7 \mathrm{~d}$ after birth.

The outcomes analyzed included the ability to transition to and wean from invasive ventilation with NAVA and NIVNAVA (including changes in vital signs, ventilator parameters, and use of sedative medications during these transitions), respiratory support at discharge, survival at discharge, the total duration of mechanical ventilation (including any form of invasive ventilation or NIV of $>2 \mathrm{~L} / \mathrm{min}$ flow), the duration of invasive ventilation, the duration of NIV, and complications that prevented the successful use of NAVA. Differences between the subjects who tolerated weaning from PC-SIMV to NAVA and those subjects who did not tolerate this approach were assessed by using a Student $t$ test for continuous variables or the Fisher exact test for categorical variables. Statistical significance was determined by $P<$ .05 .

To analyze the impact of the transition from PC-SIMV to invasive ventilation with NAVA, vital signs, respiratory parameters, blood gas values, and use of sedative medications were compared for the 24-h period before transitioning to invasive ventilation with NAVA to the 24-h period after this transition. A directional effect was specified before analysis for each outcome and one-sided $P$ values (upper tail if we hypothesized that the response after would exceed the response before; lower tail for the reverse direction) were calculated based on an exact permutation test applied to the differences (after - before). Bootstrap procedures ( $\mathrm{B}=5,000)$ were used to develop appropriate onesided 95\% confidence limits. These analyses were conducted in $\mathrm{R}$ (version 3.5.1) with the associated "boot" package2.

\section{Results}

A total of 10 subjects were admitted for surgical management of $\mathrm{CDH}$ during the review period (Table 1). The majority were boys ( 9 of 10), had left-sided $\mathrm{CDH}$ (6 of 10; 4 of 10 had right-sided $\mathrm{CDH}$ ), and had liver herniation ( 8 of 10). Birth weights ranged from 2.09 to $4.78 \mathrm{~kg}$ (mean, $3.55 \mathrm{~kg}$ ), and gestational age at birth ranged from $346 / 7$ to $405 / 7$ (mean, 39 0/7) weeks. The lung-to-head ratio (mean, 1.57 [range, 1.04-1.76]) and total fetal lung volume (mean, 30.8 [range, 18.8 to 44.0 ] $\mathrm{mL}$ ) ranges indicate that this study cohort had only mild-to-moderate risk of severe lung hypoplasia, pulmonary hypertension, or the need for extracorporeal membrane oxygenation. The majority of the subjects required patch repair of the diaphragm (6 of 10), and 4 of 10 required treatment with inhaled nitric oxide for pulmonary hypertension during the first $7 \mathrm{~d}$ after birth. Two had pulmonary sequestration (Table 1), and none were found to have a chromosome abnormality detected by comparative genomic hybridization array.

All the subjects were placed on invasive ventilation with NAVA during the postoperative period, and 7 tolerated weaning from invasive ventilation to NIV-NAVA (Table 2). The ability to successfully transition from PCSIMV to invasive ventilation with NAVA was determined by stable or improved blood gas values and the ability to wean off supplemental oxygen. The transition from PCSIMV to invasive ventilation with NAVA was successful in 8 of 10 the subjects (Table 2). Failure to tolerate the transition was evident in 2 subjects within minutes of transitioning to invasive ventilation with NAVA. Subject no. 5 was not able to be transitioned to invasive ventilation with NAVA due to an inability to reliably detect diaphragm activity indicated by a poor $\mathrm{EA}_{\mathrm{di}}$ signal. The cause of the inability to detect $\mathrm{EA}_{\mathrm{di}}$ in this subject was not clear; however, the high level of sedation and the presence of bilateral chylothorax may have contributed. Subject no. 7 was transitioned directly from PC-SIMV to NIV-NAVA (Table 2).

The successful transition from invasive ventilation with NAVA to NIV-NAVA was determined by stable or improved blood gas values and the ability to remain extubated until discharge. The transition from invasive ventilation with NAVA or PC-SIMV to NIV-NAVA was successful in 6 of 10 subjects (Table 2). Failure to tolerate the transition to NIV-NAVA required re-intubation within 1 to $5 \mathrm{~d}$ of the initial extubation in 4 subjects. The use of NAVA was impaired by the presence of symptomatic pleural effusion, defined as a pleural effusion that required increased ventilatory support, or chylothorax in 3 of the 4 subjects for whom transition to NIV by using NAVA failed (Table 2). Subject no. 3 tolerated the transition to invasive ventilation with NAVA but was not successfully 
Table 1. Demographic, Prenatal, and Early Postnatal Information of the Subjects With CDH Included in This Retrospective Review

\begin{tabular}{|c|c|c|c|c|c|c|}
\hline $\begin{array}{l}\text { Subject } \\
\text { No. }\end{array}$ & $\begin{array}{l}\text { Sex; BW (kg); } \\
\text { GA (wk) }\end{array}$ & $\begin{array}{l}\text { CDH Side; Patch; } \\
\text { Surgery DOL }\end{array}$ & $\begin{array}{c}\text { Liver } \\
\text { Herniation }\end{array}$ & $\begin{array}{l}\text { Prenatal Measurements: LHR; } \\
\text { TLFV (mL); O/E (\%) }\end{array}$ & Additional Information & $\begin{array}{l}\text { PH Treatment } \\
\quad<7 \text { DOL }\end{array}$ \\
\hline 1 & $\mathrm{M} ; 2.09 ; 346 / 7$ & Right; yes; 9 & Yes & $1.4-1.5 ; 19.8 ; 22$ & Possible coarctation of aorta & No \\
\hline 2 & $\mathrm{M} ; 3.63 ; 391 / 7$ & Left; yes; 8 & Yes & $1.68 ; 39.5 ; 60$ & & No \\
\hline 3 & $\mathrm{M} ; 3.9 ; 40$ 0/7 & Right; no; 8 & Yes & $2.76 ; \mathrm{N} / \mathrm{A} ; \mathrm{N} / \mathrm{A}$ & $\begin{array}{l}\text { Hepatopericardial fusion, right } \\
\text { pulmonary sequestration }\end{array}$ & Yes \\
\hline 4 & $\mathrm{M} ; 3.01 ; 391 / 7$ & Left; no; 8 & No & $1.04 ; 23.7 ; 29.8$ & & Yes \\
\hline 5 & $\mathrm{M} ; 3.04 ; 391 / 7$ & Left; yes; 10 & Yes & $1.30 ; 29.2 ; 55$ & & No \\
\hline 6 & M; $2.92 ; 396 / 7$ & Left; yes; 11 & No & None & Postnatal diagnosis & Yes \\
\hline 7 & $\mathrm{M} ; 4.34 ; 386 / 7$ & Right; no; 5 & Yes & $1.29 ; 29.2 ; 55$ & $\begin{array}{l}\text { Possible coarctation of aorta, } \\
\text { single umbilical artery, } \\
\text { interrupted IVC }\end{array}$ & No \\
\hline 8 & $\mathrm{M} ; 4.14 ; 405 / 7$ & Right; yes; 8 & Yes & None & Postnatal diagnosis & Yes \\
\hline 9 & F; $3.67 ; 400 / 7$ & Left; yes; 3 & Yes & N/A; $3.4 ; 41.7$ & Left pulmonary sequestration & No \\
\hline 10 & $\mathrm{M} ; 4.78 ; 392 / 7$ & Left; no; 4 & Yes & $1.5-1.7 ; 44.0 ; 68$ & & No \\
\hline
\end{tabular}

$\overline{\mathrm{CDH}}=$ congenital diaphragmatic hernia; $\mathrm{BW}=$ birth weight; $\mathrm{GA}=$ gestational age in weeks at birth; $\mathrm{DOL}=$ day of life; LHR $=$ lung-to-head ratio; TFLV = total fetal lung volume; $\mathrm{O} / \mathrm{E}=$ percent observed to expected total fetal lung volume; $\mathrm{PH}=$ pulmonary hypertension; $\mathrm{N} / \mathrm{A}=$ not available; IVC = inferior vena cava.

extubated due to persistent liver herniation, hepatopericardial fusion, and right pulmonary sequestration that could not be surgically repaired and caused compression of the right main bronchus (Table 2). Subject no. 9 was weaned from PCSIMV to invasive ventilation with NAVA and then to NIVNAVA but developed symptomatic pleural effusion that required re-intubation and subsequent management with PCSIMV (Table 2).

A comparison of the 6 subjects who successfully underwent the transition from PC-SIMV to invasive ventilation with NAVA and then to NIV-NAVA with the 4 subjects who did not tolerate this approach is shown in Table 3. There was no difference in the sex, gestational age at birth, birth weight, side of the $\mathrm{CDH}$, need for patch repair of the diaphragm, presence of liver herniation, lung-to-head ratio, total fetal lung volume, or the need for treatment of pulmonary hypertension between these groups. Factors associated with the unsuccessful use of NAVA included symptomatic pleural effusion or chylothorax $(P=.03)$, pulmonary sequestration (subject nos. 3 and 9), and the inability to reliably detect diaphragm activity (subject no. 5). The average duration of mechanical ventilation in patients who tolerated NAVA was 29.7 (median , 31.5 [range, 16-40]) d compared with 64 (median, 38.5 [range, 10-169]) d in the subjects who did not tolerate NAVA (Table 3). The total duration of invasive mechanical ventilation was $20.3 \mathrm{~d}$ for the subjects who tolerated NAVA (median, 23 [range, 928] d) compared with 57.3 (median, 6.5 [range, 7-169]) d in the subjects who did not tolerate NAVA (Table 3).

To investigate the impact of NAVA, we compared vital signs, ventilator parameters, blood gas values, and use of sedatives during the 24-h period before the transition from PC-SIMV to invasive ventilation with NAVA with those recorded during the 24-h period after this transition for the 6 subjects who were successfully managed by using this approach (Fig. 2). Although no statistically significant differences were identified in vital signs, blood gas parameters, or use of sedating medications, significant decreases in mechanical ventilation and supplemental oxygen were found after this transition. Reductions were noted for PIP $(P=.02)$ and $\overline{\mathrm{P}}_{\mathrm{aw}}(P=.047)$ (Fig. 2$)$. On average, PIP was reduced by $3.8 \mathrm{~cm} \mathrm{H}_{2} \mathrm{O}$ (minimum of $2.2 \mathrm{~cm} \mathrm{H}_{2} \mathrm{O}$ ), whereas $\overline{\mathrm{P}}_{\mathrm{aw}}$ was reduced by $1.3 \mathrm{~cm} \mathrm{H}_{2} \mathrm{O}$ (minimum of $0.4 \mathrm{~cm} \mathrm{H}_{2} \mathrm{O}$ ) after transitioning to invasive ventilation with NAVA. The $\mathrm{F}_{\mathrm{IO}_{2}}$ was reduced from 0.35 to $0.28(P=.02)$ after transitioning to invasive ventilation with NAVA. Although continuous infusions of sedating medications were weaned during the transition from PC-SIMV to invasive ventilation with NAVA, no statistically significant differences were noted in the average dose of narcotic $(P=.06)$ or benzodiazepine $(P=.47)$ infusions or in the frequency that these medications were given on an as-needed basis $(P=.25$ for narcotic mediations and $P=.25$ for benzodiazepine medications).

\section{Discussion}

Recent changes in treatment have led to improved survival among neonates with $\mathrm{CDH} .{ }^{8}$ Protecting against ventilator-associated lung injury is a major goal that may improve long-term survival and respiratory function in neonates with $\mathrm{CDH}$. At our institution, we developed a standardized approach for the management of neonates with $\mathrm{CDH}$ to decrease variation in care (Fig. 1). Given recent reports that demonstrate the potential benefit of using NAVA and our experience by using this mode of ventilation in both preterm 


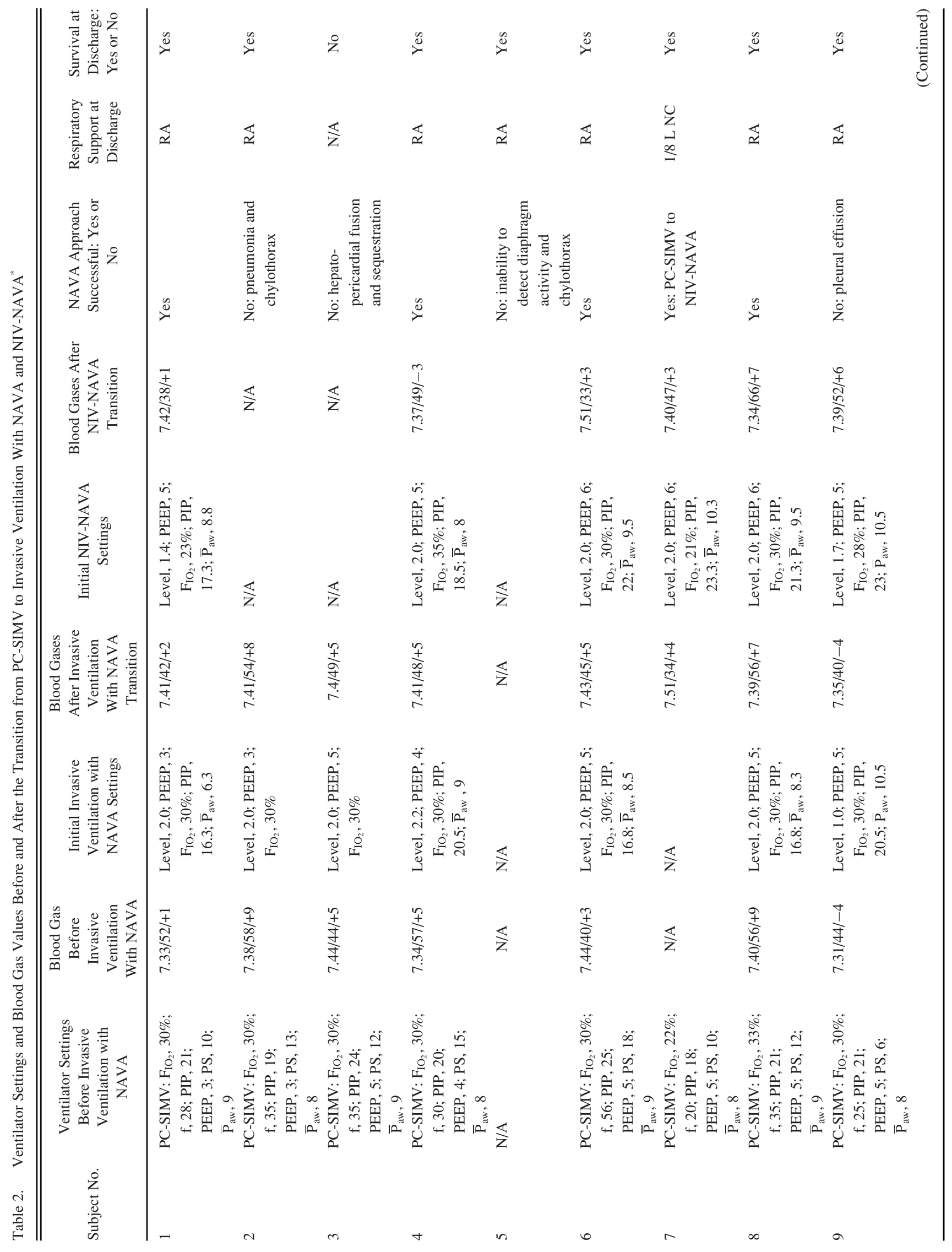




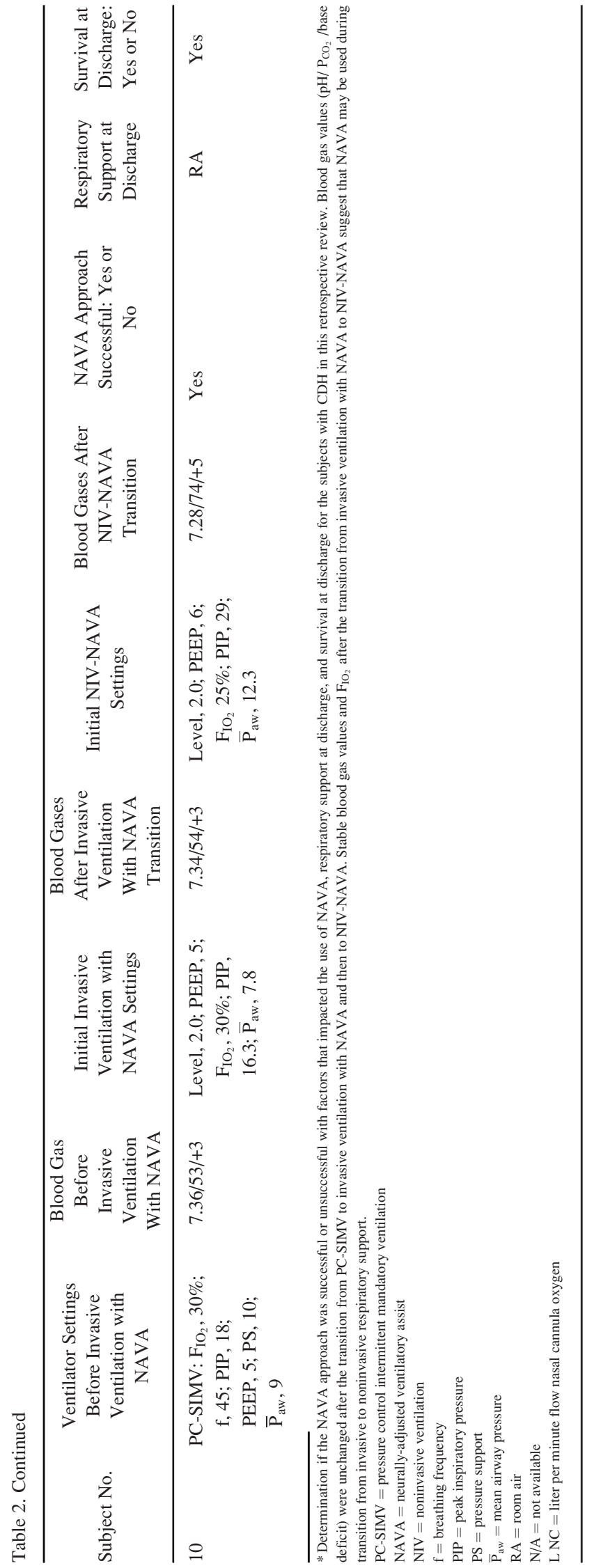

neonates and term neonates recovering from surgery, our approach encourages the use of NAVA during the transition from invasive ventilation to NIV. In this retrospective review, we demonstrated that the use of NAVA is possible during this critical transition in neonates with $\mathrm{CDH}$ who are recovering from surgical repair of the diaphragm. To the best of our knowledge, this is the first study to report the use of NAVA during the transition from invasive to NIV support in this patient population. We found that the transition from PC-SIMV to invasive ventilation with NAVA was associated with decreased PIP, $\overline{\mathrm{P}}_{\mathrm{aw}}$, and $\mathrm{F}_{\mathrm{IO}_{2}}$. Use of NAVA was impaired by the presence of symptomatic pleural effusion or chylothorax. These results are encouraging; however, this study was limited by its small size and retrospective nature, and by focusing only on short-term outcomes. A prospective randomized controlled trial will be necessary to make more definitive conclusions and determine if the use of NAVA improves the long-term survival and respiratory function of neonates with $\mathrm{CDH}$.

Despite potential advantages, there are limited data that demonstrate the benefits and risks of NAVA in neonates with $\mathrm{CDH}$. In preterm neonates, the use of NAVA has been demonstrated to decrease PIP, improve patient-ventilator synchrony, and decrease respiratory muscle work of breathing when compared with pressure targeted ventilation. $^{9,10}$ In neonates with $\mathrm{CDH}$, the transition from PCSIMV to invasive ventilation with NAVA has been correlated with decreased PIP, $\mathrm{F}_{\mathrm{IO}_{2}}, \mathrm{P}_{\mathrm{aCO}_{2}}$, and alveolar-arterial oxygen gradient $\left(\mathrm{A}-\mathrm{aDO}_{2}\right) .{ }^{5}$ Analysis of our data supported this conclusion and demonstrated that the use of NAVA in patients recovering from $\mathrm{CDH}$ repair is associated with decreases in PIP, $\overline{\mathrm{P}}_{\mathrm{aw}}$, and $\mathrm{F}_{\mathrm{IO}_{2}}$. Furthermore, analysis of our data demonstrated that blood gas values and the need for supplemental oxygen remained stable after the transition from invasive ventilation with NAVA to NIV-NAVA (Table 2), which suggested that the use of NAVA is effective during the transition from invasive to noninvasive respiratory support. Overall analysis of these data suggested that NAVA may decrease exposure to mechanical ventilation and, therefore, decrease ventilator-associated lung injury in patients with $\mathrm{CDH}$. A prospective randomized controlled trial that compares NAVA with conventional pressure or volumecontrolled ventilation would help confirm these preliminary data and determine if the use of NAVA is associated with decreased duration of invasive and total mechanical ventilation. Such a trial would also help identify important longterm benefits of using NAVA as well as potential risks, given the concerns raised by recent data that demonstrate increased driving pressure during spontaneous ventilation and its association with worsened ventilator-associated lung injury in adult patients with ARDS. ${ }^{11-13}$

Our experience was also consistent with reports that identified symptomatic pleural effusion, chylothorax, and congenital lung malformations as common barriers to 
Table 3. Comparison of the Subjects Who Tolerated Weaning from Respiratory Support by Using NAVA and NIV-NAVA (NAVA approach successful) to Those Who Did Not Tolerate This Approach (NAVA approach unsuccessful)*

\begin{tabular}{|c|c|c|c|}
\hline Variable & NAVA Approach Successful & NAVA Approach Unsuccessful & $P$ \\
\hline Subjects, $n(\%)$ & $6(60)$ & $4(40)$ & \\
\hline Boys, $n(\%)$ & $6(100)$ & $3(75)$ & .40 \\
\hline $\mathrm{BW}$, average $\pm \mathrm{SD}, \mathrm{kg}$ & $3.55 \pm 1.0$ & $3.56 \pm 0.4$ & .99 \\
\hline GA at birth, average $\pm \mathrm{SD}$, wk & $38.8 \pm 2.0$ & $39.6 \pm 0.5$ & .47 \\
\hline Left-sided CDH, $n(\%)$ & $3(50)$ & $3(75)$ & .57 \\
\hline Patch repair, $n(\%)$ & $3(50)$ & $3(75)$ & .57 \\
\hline DOL at surgery, average \pm SD & $7.5 \pm 2.6$ & $7.3 \pm 3.0$ & .91 \\
\hline Liver herniation, $n(\%)$ & $4(67)$ & $4(100)$ & .47 \\
\hline LHR, average \pm SD & $1.31 \pm 0.2$ & $1.91 \pm 0.8$ & .18 \\
\hline $\mathrm{O} / \mathrm{E}$ TFLV, average $\pm \mathrm{SD}$ & $44(21)$ & $58(29)$ & .46 \\
\hline $\mathrm{PH}$ that required treatment $<7 \mathrm{DOL}, n(\%)$ & $3(50)$ & $1(25)$ & .57 \\
\hline Symptomatic pleural effusion or chylothorax, $n(\%)$ & $0(0)$ & $3(75)$ & .03 \\
\hline Anatomic lung defects, $n(\%)$ & $0(0)$ & $2(50)$ & .13 \\
\hline Mechanical ventilation, average $\pm S D, d$ & $29.7 \pm 8.2$ & $64 \pm 71.3$ & .20 \\
\hline Invasive mechanical ventilation, average $\pm \mathrm{SD}, \mathrm{d}$ & $20.3 \pm 7.0$ & $57.3 \pm 75.1$ & .25 \\
\hline NIV, average $\pm S D, d$ & $9.3 \pm 6.7$ & $12 \pm 5.7$ & .64 \\
\hline
\end{tabular}

* No difference was observed in BW, GA at birth, side of CDH, frequency of patch repair of the diaphragm, DOL at surgery, frequency of liver herniation, estimated fetal lung size measured by LHR or total fetal lung volume, need for treatment of PH, or duration of mechanical ventilation between the group who tolerated weaning with NAVA compared with the group who did not tolerate NAVA. Common factors associated with failure of NAVA included symptomatic pleural effusion and chylothorax $(P=.03)$. Comparisons were made by using a Student $t$ test for continuous variables and the Fisher exact test for categorical variables.

$\mathrm{NAVA}=$ neurally-adjusted ventilatory assist; NIV $=$ noninvasive ventilation; $\mathrm{BW}=$ birth weight; $\mathrm{GA}=$ gestational age; $\mathrm{CDH}=$ congenital diaphragmatic hernia; $\mathrm{DOL}=$ day of life; LHR $=$ lung-tohead ratio; O/E TFLV = observed-to-expected total fetal lung volume; $\mathrm{PH}=$ pulmonary hypertension.

the successful use of NAVA in neonates with $\mathrm{CDH}^{5-7}$ Symptomatic pleural effusion and chylothorax that required escalation of respiratory support were common in our cohort, which affected $30 \%$ of the subjects. Management decisions with regard to the increase of respiratory support for subjects with symptomatic pleural effusion or chylothorax was left to the discretion of the supervising attending physician. In each case, these subjects were treated by converting to PC-SIMV. It remains unclear if a large pleural effusion or chylothorax interferes with NAVA or the ability to detect an adequate $\mathrm{EA}_{\mathrm{di}}$ signal. Interestingly, after surgical repair of the diaphragm, all the subjects had accumulation of fluid in the ipsilateral hemithorax as is typical in patients with $\mathrm{CDH}$. Despite the presence of this fluid, an adequate $\mathrm{EA}_{\mathrm{di}}$ signal was detectable in the majority of the subjects within 24-72 h of surgery.

Furthermore, subject no. 5 was unable to transition to invasive ventilation with NAVA due to an inability to detect an adequate $\mathrm{EA}_{\mathrm{di}}$ signal, which was unique to this case. Subject no. 5 had isolated left-sided $\mathrm{CDH}$ with liver herniation and reassuring estimated fetal lung volume (observed-to-expected lung volume ratio, $55 \%$ ), and required patch repair of the diaphragm. The presence of liver herniation and the need for patch repair of the diaphragm were not associated with failure to transition to invasive ventilation with NAVA in this cohort (Table 3). Because of the need to escalate respiratory support after surgery and the subsequent need to place a chest tube, this subject was treated with higher than typical doses of sedative medications, which may have impaired the ability to detect an adequate $\mathrm{EA}_{\mathrm{di}}$ signal.

In addition to decreasing exposure to invasive mechanical ventilation, another important goal is to decrease the need for sedative medications. Neonates with CDH typically require treatment for pain and agitation after birth and $\mathrm{CDH}$ surgical repair. Although treatment is warranted during these phases of care, use of these medications is correlated with iatrogenic withdrawal symptoms and prolonged hospitalization. ${ }^{14}$ An early transition to spontaneous respiratory support and extubation may decrease the need for treatment with sedating medications and reduce the risk of neurologic impairment associated with recurrent exposure to these mediations. ${ }^{14}$ Because NAVA relies on the presence of $\mathrm{EA}_{\mathrm{di}}$, treatment with narcotic or sedating medications that suppress the respiratory drive may hinder the effectiveness of NAVA. ${ }^{9,15}$ Analysis of our data demonstrated that neonates with $\mathrm{CDH}$ who transitioned from PC-SIMV to invasive ventilation with NAVA could tolerate routine weaning of sedating medications; however, it remains unclear if use of NAVA facilitates more rapid weaning. The experience of staff members and their level of comfort with NAVA are important factors that can limit its use and effectiveness. Servo (Maquet, Solna, Sweden) is the only ventilator that is compatible with NAVA and requires specialized training for its use. Furthermore, the catheters used to detect the $\mathrm{EA}_{\mathrm{di}}$ signal cost $\$ 200$ each. Therefore, the impact of short-term improvements in respiratory parameters, as 
NAVA to Facilitate Extubation in NeOnates With CDH

\begin{tabular}{|c|c|c|c|}
\hline Variable & Before NAVA & After NAVA & $P$ \\
\hline Heart rate \pm SD & $154 \pm 13$ & $155 \pm 12$ & .67 \\
\hline Breathing frequency $\pm S D$ & $57 \pm 9$ & $52 \pm 11$ & .17 \\
\hline Pre-ductal saturation \pm SD & $98 \pm 1$ & $99 \pm 1$ & .27 \\
\hline $\mathrm{pH} \pm \mathrm{SD}$ & $7.34 \pm 0.07$ & $7.38 \pm 0.01$ & .13 \\
\hline $\mathrm{P}_{\mathrm{CO}_{2}} \pm \mathrm{SD}$ & $52 \pm 7$ & $50 \pm 6$ & .30 \\
\hline Morphine infusion dose \pm SD & $0.07 \pm 0.04$ & $0.06 \pm 0.04$ & .06 \\
\hline $\begin{array}{c}\text { Number of narcotic PRN } \\
\text { doses } \pm \text { SD }\end{array}$ & $3.3 \pm 1.9$ & $2.8 \pm 3.4$ & .25 \\
\hline Midazolam infusion dose \pm SD & $0.07 \pm 0.07$ & $0.05 \pm 0.06$ & .47 \\
\hline $\begin{array}{c}\text { Number of benzodiazepine } \\
\text { PRN doses } \pm \text { SD }\end{array}$ & $3.8 \pm 2.0$ & $3.0 \pm 1.9$ & .25 \\
\hline
\end{tabular}
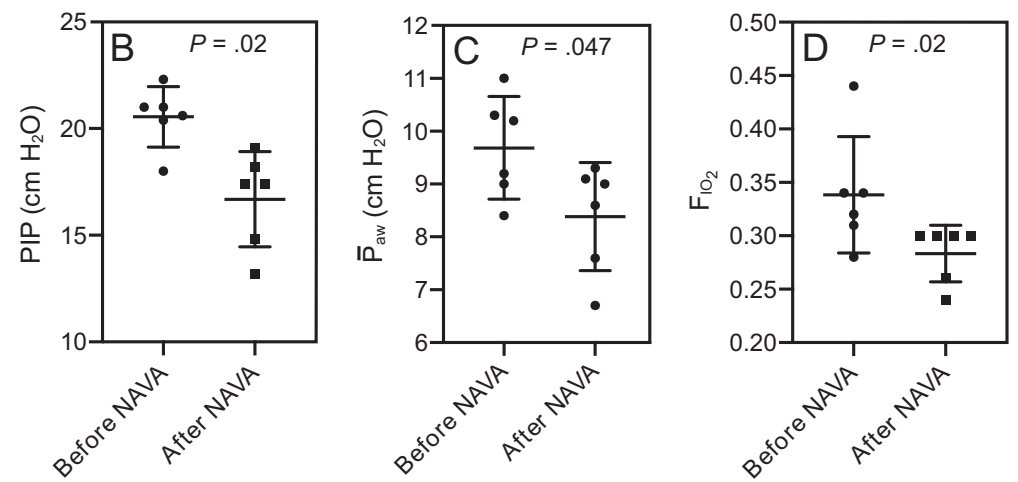

Fig. 2. A: Comparison of the $24 \mathrm{~h}$ before the transition from pressure control intermittent mandatory ventilation (PC-SIMV) to invasive ventilation with neurally-adjusted ventilatory assist (NAVA) to the $24 \mathrm{~h}$ after this transition in the subjects who tolerated being weaned from respiratory support when using NAVA. Average vital signs, blood gas values, and use of sedating medications were similar before and after the transition to invasive ventilation with NAVA (I-NAVA). B-D: Peak inspiratory pressure (PIP) $(P=.02)$, mean airway pressure $\left(\overline{\mathrm{P}}_{\mathrm{aw}}\right)(P=.047)$, and $\mathrm{F}_{1 \mathrm{I}_{2}}(P=.02)$ were decreased after the transition to I-NAVA ( $P<.5)$. Morphine and midazolam infusion doses are reported as $\mathrm{mg} / \mathrm{kg} / \mathrm{h}$. Narcotic as-needed medications were morphine or fentanyl; benzodiazepine as-needed medications were midazolam or lorazepam and are reported as the number of doses given during each 24-h period.

observed in this retrospective review, on the duration of ventilation and hospitalization should be considered against the cost of NAVA catheters and Servo ventilators.

Also, our data were limited to a small retrospective case series with a predominance of male subjects with mild-tomoderate risk of severe lung hypoplasia, a need for extracorporeal membrane oxygenation, or death. Additional limitations included the lack of data investigating the impact of NAVA on the cost and stay for patients supported with NAVA. Although we adopted a neonatal $\mathrm{CDH}$ management guideline, medical decision-making was left to provider discretion, which may have introduced additional bias. Despite these limiting factors, we demonstrated that both invasive ventilation with NAVA and NIV-NAVA were feasible in neonates with pulmonary hypertension requiring vasodilator therapy, left- and right-sided
$\mathrm{CDH}$, and liver herniation before surgery, and in patients who required patch repair of the diaphragm.

NAVA is also feasible in neonates with $\mathrm{CDH}$ of various gestational ages (34 6/7 to 40 5/7 weeks) and birth weights ( 2.09 to $4.78 \mathrm{~kg}$ ) as demonstrated in our cohort. Our data should be interpreted cautiously until the use of NAVA in patients with $\mathrm{CDH}$ can be more thoroughly evaluated in a multi-center, prospective, randomized controlled trial. Such a trial would be beneficial to investigate both the risks and impact on survival, duration of mechanical ventilation, respiratory requirements at discharge, stay, duration and extent of pain control and sedation medications, progression of oral feedings, and long-term respiratory outcomes in neonates supported with NAVA compared with conventional ventilation. 


\section{NAVA to Facilitate Extubation in NeOnates With CDH}

\section{Conclusions}

Our retrospective case series demonstrated that invasive ventilation with NAVA and NIV-NAVA could be used during the transition from invasive ventilation to NIV in neonates with $\mathrm{CDH}$. The transition to NAVA was associated with a decrease in PIP, $\overline{\mathrm{P}}_{\mathrm{aw}}$, and need for supplemental oxygen. Symptomatic pleural effusion and chylothorax were identified as common barriers that limited the successful use of NAVA during the postoperative weaning phase of respiratory support. Further investigation with a randomized controlled trial is warranted to determine if NAVA can reduce ventilator-associated lung injury in patients with $\mathrm{CDH}$ and improve their long-term outcomes.

\section{ACKNOWLEDGMENTS}

We thank the members of the UW Health Clinical Care Guideline Committee for their help developing and implementing the $\mathrm{CDH}$ Management Guideline. We thank the nurses, respiratory therapists, and physicians in the American Family Children's Hospital neonatal ICU and pediatric ICU for their help with implementing the use of NAVA in patients with CDH. Finally, we thank Michael Lasarev from the University of Wisconsin-Madison Department of Biostatistics and Medical Informatics for his help with our statistical analysis.

\section{REFERENCES}

1. Badillo A, Gingalewski C. Congenital diaphragmatic hernia: treatment and outcomes. Semin Perinatol 2014;38(2):92-96.

2. Guevorkian D, Mur S, Cavatorta E, Pognon L, Rakza T, Storme L. Lower distending pressure improves respiratory mechanics in congenital diaphragmatic hernia complicated by persistent pulmonary hypertension. J Pediatr 2018;200:38-43.

3. Mally PV, Beck J, Sinderby C, Caprio M, Bailey SM. Neural breathing pattern and patient-ventilator interaction during neurally adjusted ventilatory assist and conventional ventilation in newborns. Pediatr Crit Care Med 2018;19(1):48-55.
4. Alander M, Peltoniemi O, Pokka T, Kontiokari T. Comparison of pressure-, flow-, and NAVA-triggering in pediatric and neonatal ventilatory care. Pediatr Pulmonol 2012;47(1):76-83.

5. Gentili A, Masciopinto F, Mondardini MC, Ansaloni S, Reggiani ML, Baroncini S. Neurally adjusted ventilatory assist in weaning of neonates affected by congenital diaphragmatic hernia. J Matern Fetal Neonatal Med 2013;26(6):598-602.

6. Oda A, Lehtonen L, Soukka H. Neurally adjusted ventilatory assist can be used to wean infants with congenital diaphragmatic hernias off respiratory support. Acta Paediatr 2018;107(4):718-719.

7. Amin R, Arca MJ. Feasibility of non-invasive neurally adjusted ventilator assist after congenital diaphragmatic hernia repair. J Pediatr Surg 2019;54(3):434-438.

8. Putnam LR, Harting MT, Tsao K, Morini F, Yoder BA, Luco M, et al; Congenital Diaphragmatic Hernia Study Group. Congenital diaphragmatic hernia defect size and infant morbidity at discharge. Pediatrics 2016;138(5):e20162043.

9. Lee J, Kim H-S, Jung YH, Choi CW, Jun YH. Neurally adjusted ventilatory assist for infants under prolonged ventilation. Pediatr Int 2017;59(5):540-544.

10. Stein H, Alosh H, Ethington P, White DB. Prospective crossover comparison between NAVA and pressure control ventilation in premature neonates less than 1500 grams. J Perinatol 2013;33(6):452-456.

11. Brochard L, Slutsky A, Pesenti A. Mechanical ventilation to minimize progression of lung injury in acute respiratory failure. Am J Respir Crit Care Med 2017;195(4):438-442.

12. van Haren F, Pham T, Brochard L, Bellani G, Laffey J, Dres M, et al.; Large observational study to UNderstand the Global impact of Severe Acute respiratory FailurE (LUNG SAFE) Investigators. Spontaneous breathing in early acute respiratory distress syndrome: insights From the Large Observational Study to UNderstand the Global Impact of Severe Acute Respiratory FailurE Study. Crit Care Med 2019;47(2):229-238.

13. Yoshida T, Amato MBP, Kavanagh BP, Fujino Y. Impact of spontaneous breathing during mechanical ventilation in acute respiratory distress syndrome. Curr Opin Crit Care 2019;25(2):192-198.

14. Ng E, Taddio A, Ohlsson A. Intravenous midazolam infusion for sedation of infants in the neonatal intensive care unit. Cochrane Database Syst Rev 2017(1):CD002052.

15. Rhoney DH, Murry KR. National survey of the use of sedating drugs, neuromuscular blocking agents, and reversal agents in the intensive care unit. J Intensive Care Med 2003;18(3):139-145. 